\title{
Isotopic characterization of the benthic iron flux in the Changjiang Estuary
}

\author{
ZHIWEI HE ${ }^{1}$, SHOUYE YANG ${ }^{1 *}$, ERGANG LIAN ${ }^{1}$, PINGHE \\ $\mathrm{CAI}^{2}$, RUIFENG ZHANG ${ }^{3}$
}

${ }^{1}$ State Key Laboratory of Marine Geology, Tongji University, Shanghai 200092, China

${ }^{2}$ State Key Laboratory of Marine Environmental Science, Xiamen University, Xiamen 361005, China

${ }^{3}$ School of Oceanography, Shanghai Jiao Tong University, Shanghai 200030, China

(*correspondence: syyang@tongji.edu.cn)

Iron $(\mathrm{Fe})$ is an essential micronutrient in the ocean, and plays a key role in regulating the magnitude and dynamics of ocean primary production $[1,2]$. The last several decades have seen numerous studies attempting to constrain its biogeochemical cycling, sources, and sinks in the global ocean. Iron isotopes have emerged as a powerful tool to quantify the oceanic Fe sources and cycle [3]. Despite the significant terrigenous iron input to ocean [3], the benthic flux of $\mathrm{Fe}$ and $\mathrm{Fe}$ isotopes from global estuaries remains poorly constrained.

Benthic fluxes of $\mathrm{Fe}$ revealed by ${ }^{224} \mathrm{Ra} /{ }^{228} \mathrm{Th}$ disequilibria can be as high as $\sim 100 \mathrm{mmol} \mathrm{m}^{-2} \mathrm{~d}^{-1}$ from China's estuaries [4], largely beyond the the previous estimation based on the traditional incubation and molecular diffusion methods. In this context, there is an urgent need to characterize the isotopic signature of Fe released from estuarine sediments in order to fingerprint its biogeochemical impacts in coastal ecosystems. Here we present the first pore water and solid phase $\mathrm{Fe}$ isotope data collected on a transect across the Changjiang (Yangtze R.) Estuary and adjacent shelf with bottom water oxygen concentrations ranging from 51 to 181 $\mu \mathrm{M}$. Our findings reveal that the amount and isotopic composition of $\mathrm{Fe}$ exporting to the ocean respond in a specific manner to the benthic redox conditions. To address the causes for the spatial pattern in the magnitude of benthic $\mathrm{Fe}$ flux and associated Fe isotopic signature, we discuss the influences of the availability of organic matter and reducible $\mathrm{Fe}$ oxides as well as bioturbation and physical reworking processes. This study implies that the iron isotopes can constrain the benthic Fe flux from global estuaries and thus contribute to Fe budget quantification in coastal oceans.

[1] Boyd and Ellwood (2010) Nature Geosci. 3, 675-682. [2] Tagliabue et al. (2017) Nature 543, 51-59. [3] Conway\&John (2014) Nature 511, 212-215. [4] Shi et al. (2019) Geochim. Cosmochim. Acta 260, 49-61. 